\title{
Computer Programming Object
}

National Cancer Institute

\section{Source}

National Cancer Institute. Computer Programming Object. NCI Thesaurus. Code C45974.

In object-oriented programming, an object is a self-contained entity that consists of both data and procedures to manipulate the data. 